\title{
Distal tibiofibular synostosis in a Nigerian: a case report
}

\author{
Olatunde OWOEYE* and Oladayo Sunday OYEDUN \\ ${ }^{I}$ Department of Anatomy, College of Medicine, University of Ibadan, Ibadan, Nigeria. \\ *Corresponding Author, E-mail: o.owoeye@mail.ui.edu.ng; oowoeye2001@yahoo.com; \\ Tel: +234-8033239973; Fax: 234-2-8103043
}

\begin{abstract}
Tibiofibular synostosis (TFS) is a rare cause of recurrent anterior shin pain encountered in sport injuries following the ossification of the tibiofibular membrane. Awareness of distal TFS prevents misinterpretation of $\mathrm{X}$-rays and enables efficient management of individuals with post traumatic TFS. Following maceration of a dissected male cadaver, we came across a tibia and fibula with $29.09 \times 11.83 \times 22.28 \mathrm{~mm}$ ossified distal tibiofibular membrane. The ossified membrane had a $26.16 \mathrm{~mm}$ long, inferomedially directed groove on its posterior surface. X-ray of the bones showed an oblique fracture in the distal end of the shaft of fibula which is suggestive of post traumatic tibiofibular synostosis (TFS). Knowledge of distal TFS is important in resolving the puzzle of chronic shin pain of unknown origin and in accurate diagnosis of causes of ankle deformity and malformations.
\end{abstract}

() 2015 International Formulae Group. All rights reserved.

Keywords: Tibiofibular synostosis, post-traumatic ossification, chronic shin pain.

\section{INTRODUCTION}

Tibiofibular synostosis (TFS) is one of the unusual causes of anterior shin pain encountered in sport injuries. This condition is characterized by the ossification of the aponeurotic fibers of the tibiofibular membrane (Williams et al., 2008). The tibiofibular membrane extends between the interosseous crests of tibia and fibula, thus separating the anterior leg muscles from the posterior (Williams et al., 2008). The lower part of this membrane has openings for the passage of anterior peroneal vessels (Williams et al., 2008). Based on location, TFS can be classified as distal and proximal. Proximal TFS has a congenital etiology and has been reported to occur in association with angular deformity, leg length discrepancy, multiple hereditary exostoses and peroneaal mononeuropathy (Nishikawa et al., 2003; Bozcurt et al., 2004). Distal TFS is usually acquired, occurring in tibiofibular fractures or as complication of orthopaedic procedures (Frick et al., 2001). There are few reports on tibiofibular synostosis in the Nigerian population. We present a rare case of distal TFS in Nigerian tibia and fibula.

\section{CASE REPORT}

Following maceration of a dissected male cadaver in the Department of Anatomy, University of Ibadan, Nigeria, we came across a dried adult left tibia and fibula with ossified tibiofibular membrane (Figures 1A\&1B). This 
ossified membrane measured $29.09 \mathrm{~mm}$ in length, $11.83 \mathrm{~mm}$ in breadth and $22.28 \mathrm{~mm}$ in thickness. There was no associated ossification of the distal tibiofibular joint.

The posterior surface of the ossified tibiofibular membrane had a shallow, inferomedially directed groove (Figure 1B). This groove measured $26.16 \mathrm{~mm}$ in length. The ossified tibiofibular membrane had small openings for the passage of anterior peroneal vessels (Figure 2A). The tibia length, measured as a distance from the top of intercondylar eminence to the inferior end of medial malleolus was $42.5 \mathrm{~cm}$. The fibular length, measured as a distance from the top of styloid process to the inferior end of lateral malleolus was $40.2 \mathrm{~cm}$. Plain radiograph (X ray) of the bones showed an oblique fracture in the distal end of the shaft of fibula, adjacent to the synostosis (Figure 2B). However, no medullary cavity shadow was present in the ossified membrane (Figure 2B) and the age of the cadaver was unknown.
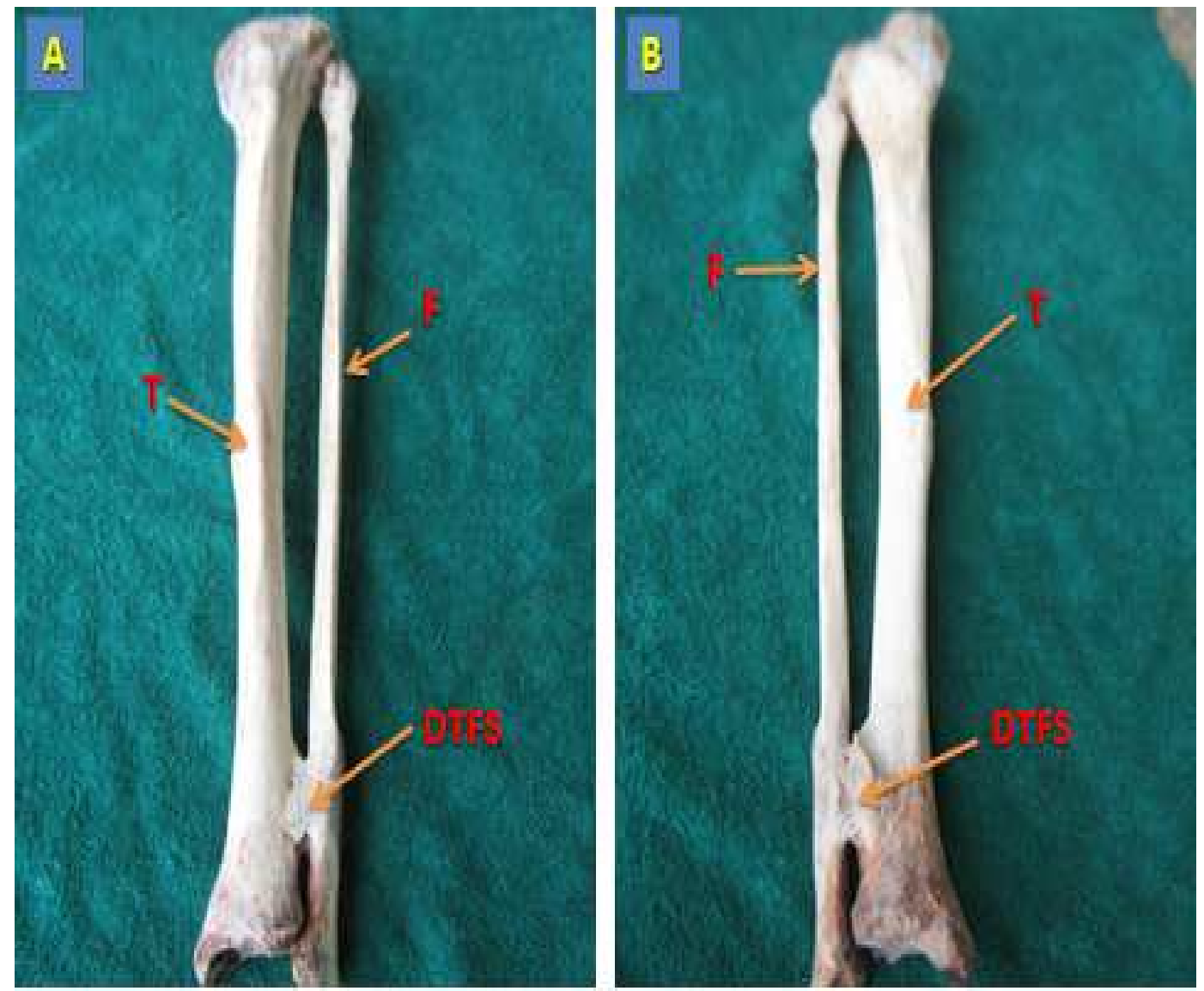

Figure 1: Photographs of the left tibia and fibula showing Distal Tibiofibular Synostosis. (1A-anterior view, 1B-Posterior view). T- Tibia, F- fibula, DTFS- distal tibiofibular synostosis. 

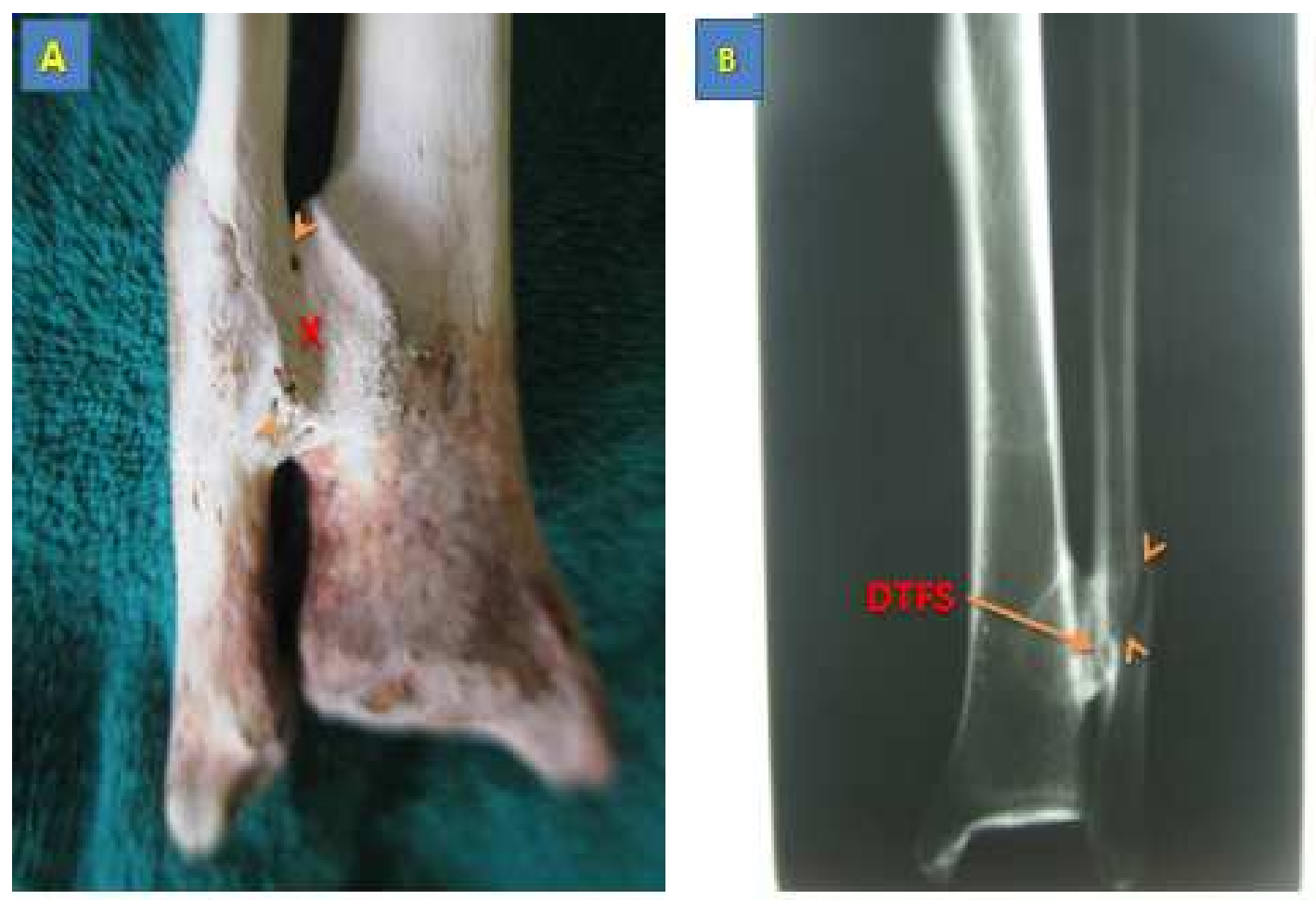

Figure 2: Photograph of the distal end of the posterior surface of the left tibia and fibula showing a shallow groove $(\mathrm{X})$ and openings for anterior tibial vessels (arrowheads) in the ossified membrane (A); Radiograph of the left tibia and fibula showing oblique fracture of the distal fibula (B). (DTFSDistal Tibiofibular synostosis).

\section{DISCUSSION}

Synostosis is a component of a wide spectrum of extraskeletal calcification termed heterotopic ossification. Other components of heterotopic ossification include ossification of muscle tendons (Rider's bone, Shooter's bone, Fencer's bone) and muscle bulk (myositis ossificans). Distal TFS has been reported in athletes following repeated ligament sprain of the ankle (Williams et al., 2007). Iatrogenic causes have been described following syndesmotic screw insertion and removal (Miller et al., 2010). Though the exact mechanism of ossification in post traumatic distal TFS is not fully understood, ossification of the hematoma tracking on the distal tibiofibular membrane has been suggested (James et al., 2007). The post traumatic etiology was upheld by Fu et al. (2003), who reported tibiofibular synostosis in a 21 year old soldier following a left ankle sprain sustained 5 years prior to presentation. Tibiofibular synostosis has also been reported in fractures especially in stress fractures (Horst and Nunley, 2004).

The present case report is post traumatic in view of the distal shaft fracture of fibula and the distal location of the synostosis. Like in other reported cases, the individual in his life time is likely to have suffered chronic shin pain and impaired ankle function. Fu et al. (2003) suggested that distal TFS prevents the descent of the fibula during the mid-stance and pre-swing phase of gait .Distal TFS may cause compression of the anterior peroneal vessels which run through perforations in the lower part of distal tibiofibular membrane. Distal post traumatic synostosis can be 
effectively managed by resecting the syostosis along with the surrounding pathological bones (James et al., 2007).

\section{Conclusion}

Knowledge of distal TFS is important in resolving the puzzle of chronic shin pain of unknown origin and in accurate diagnosis of causes of ankle deformity and malformations. Awareness of distal TFS prevents misinterpretation of X-rays and enables efficient management of individuals with post traumatic TFS.

\section{ACKNOWLEDGEMENTS}

Authors gratefully acknowledge Mrs. M. Okaku, Radiological Unit, University Health Center, University of Ibadan, Nigeria, for the technical assistance in the preparation of the radiological films.

\section{REFERENCES}

Bozkurt M, Dogan M, Turanli S. 2004. Osteochondroma leading to proximal tibiofibular synostosis as a cause of persistent ankle pain and lateral knee pain. Knee Surg.Sp. Traum. Arthro., 12(2): 152-154.

Frick SL, Shoemaker S, Mubarak SJ. 2001. Altered fibula growth patterns after tibiofibular synostosis in children. $J$ Bone Joint SurgAm., 83-A(1 ): 247-254.

$\mathrm{Fu}$ JH, Hwang CC, Chao TH. 2003. Tibiofibular synostosis in a military soldier. J. Med. Sci., 23(2): 135-138.

HorstF, Nunley JA. 2004. Tibiofibular Synostosis Stress Fracture: A Case Report. Foot \& Ankle International, 25(7): 507-509.

James SH, Carpenter EC, Fairclough JA. 2007. Tibiofibular synostosis in a professional football player. J. Bone. Joint. Surg., 89-B(1):109-11.

Miller AN, Paul O, Boraiah S, Parker RJ, Helfet DL, Lorich DG. 2010. Functional outcomes after syndesmotic screw fixation and removal. Journ. Ortho. Traum., 24(1): 12-16.

Nishikawa T, Yamamoto T, Yoshiya S. 2003. Intermittent peroneal mono-neuropathy due to proximal tibiofibular synostosis. $J$. Neurol., 250(3): 352-353.

Williams GN, Jones MH, Amendola A. 2007. Syndesmotic Ankle Sprains in Athletes. Am. Jour. Sp. Med., 35(7): 1197-1207.

Williams P, Warwick R, Dyson M, Bannister LH, Standring S. 2008. Gray's Anatomy. The Anatomical Basis of Clinical Practice, $\quad\left(40^{\text {th }}\right.$ edn). Churchill Livingstone Elsevier: London; 712-713. 